I.

\title{
Das klinische Gesamtbild der endothorakalen Drüsen- und Lungenhilustuberkulose der Er- wachsenen.
}

Von

Dr. H. Philippi,

Chefarzt des Internationalen Sanatoriums Davos-Dorf.

Obwohl durch die ausgezeichneten Arbeiten von Biedert (1), Heubner (2), De la Camp (3), Brecke (4), Köhler (5), $\mathrm{Kräm}$ er (6), H u guenin (7, 8), Da utwit $z$ (9) und anderen das klinische Bild der endothorakalen Drüsentuberkulose schon recht gut abgegrenzt wurde, fehlt es doch an einer kritischen Zusammenstellung aller hierher gehörigen klinischen Symptome, inklusive des doch recht eigentümlichen Blutbildes. Fs mag dies mit der Grund sein, dass diese Diagnosie nur sehr selten gestellt wird, auch in ganz typischen. Fällen.

Vor allem begegnet man immer wieder dem Einwand, dass die Symptome doch zu unbestimmt und nicht eindeutig genug seien.

Nur muss zugegeben werden, dass man erst aus einer Summe von klinischen Erscheinungen heraus die Diagnose auf eine Erkrankung der inneren Thoraxdrüsen mit einiger Sicherheit stellen kann, wodurch man dann bestimmt werden soll, die vermutete Diagnose durch weitere Untersuchungen, speziell durch Röntgenaufnahmen und Blutuntersuchungen zu befestigen.

Dabei ist aber wohl im Auge zu behalten, dass, wie gerade auch die Röntgenaufnahmen zeigen, es sich nur höchst selten um reine Drüsenprozesse handelt, sondern, dass wenigstens bei Erwachsenen sozusągen immer eine mehr oder weniger ausgesprochene Erkrankung des umgebenden Hilusgewebes, d. h. in Form peribronchitischer und auch infiltrativer Prozesse des angrenzenden Lungengewebes, vorliegt. 
Je nach dem Grad der Ausbreitung des tuberkulösen Prozesses werden auch die Symptome eine gewisse Beeinflussung erfahren; das Gesamtbild der klinischen Erscheinungen ist aber für die Tuberkulose der betreffenden Organe immer charakteristisch.

Auf die genauere Schilderung der diesbezüglichen anatomischen und pathologisch-anatomischen Verhältnisse muss an dieser Stelle verzichtet werden. Ẽs sei auf die einschlägige. Arbeit von Köhler 1. c. sowie auf die folgende Studie von meinem früheren Assistenzarzt E. Schulz verwiesen.

Im folgenden sollen nun die hauptsächlichsten klinischen Erscheinungen zusammengefasst werden, die den Verdacht eines endothorakalen Drüsenprozesses nahelegen.

Es sei gleich vorausigeschickt, dass in allen unsieren Fällen, wo wir schon klinisch die Diagnose einer Hiluserkrankung stellten, wir auch röntgenologisch unsere Diagnose bestäitigen konnten.

In einer zweiten Arbeit wird F. Sc hulz über seine, auf meine Veranlassung gemachten Blutuntersuchungen bei einigen Fällen von endothorakaler Drüsentuberkulose berichten und zeigren, dass uns solche Untersuchungen ein ferneres, wichtiges Dokument zur Vervoliständigung unserer Diagnose liefern können, gerade, was die eigentlichen, aktiven Drüsenprozesse, seien es solche primärer oder sekundärer Art, anbelangt.

Ich muss nun zunächst der immer noch vielfach verbreiteten Ansicht entgegentreten, wonach solche Drüsenprozesse, wenigstens die primären, fast nur bei Kindern vorkommen sollen, wie es z. B. auch Bandelier und Roepke (10) behaupten, wenn sie sagen, dass jenseits des Pubertätsalters die primäre Bronchialdrüsentuberkulose ihre Rolle ausgespielt habe. Auch $\mathrm{Huguenin}$ (l. e. 8) bezeichnet die primäre Bronchialdrüsentuberkulose bei Erwachsenen als eine Seltenheit.

Gewiss sind solche Prozesse häufiger bei Kindern als bei Erwachsenen, stellen sie doch häufig bezüglich der klinischen Erscheinungen den ersten Akt in dem Trauerspiel einer sich entwickelnden Lungentuberkulose dar. Doch besteht für uns mit Krämer (l. c.) gar hein Zweifel, dass bei Erwachsenen die endothorakalen Drüsen $z$ u ers t erkranken können. Dabei liegen folgende Möglichkeiten vor. - Es handelt sich um eine frische Infektion, die von irgend einer Eingangspforte, und zwar in der Mehrzahl der Fälle wohl von der Lunge her sich ihren Weg in die inneren Thoraxdrüsen bahnt. Dabei sei an die ausserordentlich interessanten, noch viel zu wenig bekannten Versuche von Heymann (11) aus dem 
Flügg e schen Laboratorium erinnert, der einwandsfrei nachwies, dass bei Inhalation von mittleren Dosen von Tuberkelbazillen 3 Tage - von grossen Dosen bereits 1 Stunde - nach der Inhalation Tuberkelbazillen in den Bronchialdrüsen nachzuweisen sind. Andererseits kommt es vor, dass ein alter, latent gewordener endothorakaler Lrüsenprozess durch irgend einen Umstand, z. B. eine Zwischeninfektion nichttuberkulöser Art, allgemein schwächende Momente, ein Trauma usw. aufflackert.

Fragen wir nun zunächst nach den subjektiven Erschei $\mathrm{n} u \mathrm{n} g$ e $\mathrm{n}$, die ein beginnender endothorakaler Drüsenprozess machen kann, so ist ganz allgemein zu sagen, dass diese mit wenigen Auswahmen durchaus diejenigen einer beginnenden aktiven Tuberkulose sind, ohne auf eine besondere Lokalisation hinzuweisen. Auffallend war mir in vielen Fällen ein allgemeines Schwäch egefühl, das oft in merkwürdigem Gegensatz zu einer guten allgemeinen Ernährung stand, ebenso eine leichte geistige und körperliche Ermüdbarkeit, oft auch eine sehr labile Stim. mung.

Einige Patienten klagten auch über zeitweise auftretende Schmerzen unter dem Brustbein oder zwischen den Schulterblättern; diese Schulterblattschmerzen strahlten bei einigen zeitweise sogar bis in den Arm der befallenen Seite aus. Bei Frauen steigerten sich diese Symptome mit grosser Regelmässigkeit vor der Periode.

Manche dieser Kranken litten an ausgesprochener nervöser Dyspepsie, meist mit Superazidität und zeitweise auftretenden Magenschmerzen, die sich auch bis zu eigentlichen Magenkrämpfen steigeri: konnten, ähnlich den tabischen Krisen, worauf schon Brecke (l. c.) aufmerksam gemacht hat.

Bei einzelnen Patienten zeigte sich Neigung zu Herzklopfen und das Gefühl unregelmässiger Herztätigkeit.

Dass solche Patienten etwa auch an keuchhustenartigen, $\mathrm{Hustenparoxismen} \mathrm{leiden,} \mathrm{die} \mathrm{sich} \mathrm{bis} \mathrm{zu} \mathrm{asthmaartigen} \mathrm{An-}$ fällen steigern können, wird von verschiedenen Autoren, z. B. von $\mathrm{Brecke}$ (l. c.) angegeben; ich habe dies nur selten beobachtet. Jedenfalls scheint, was Brecke (l. c.) auch betont, mehr Vagusdruck als direkte Kompression der kranken Bronchien das Zustandekommen dieser Erscheinungen zu verursachen.

Die eigentlichen Kompressionserscheinungen durch Druck starker Drüsenschwellungen auf die anderen Nachbarorgane (grosse Gefässe, Hauptbronchien usw.), wie sie von Brecke 
(1. c.), De la Camp (l. c.), Bandelier und Roepke (l. c.) beschrieben wurden, will ich hier nicht besprechen, da dies bei Erwachsenen nur selten vorkommt. Erreichen doch diese Lymphdrüsenschwellungen bei Erwachsenen im allgemeinen verhältnismässig nur geringere Grade als bei Kindern. Ich habe bei meinen Fällen derartige ausgesprochene Kompressionserscheinungen nie beobachtet.

Ziemlich häufig klagen solche Drüsenpatienten, über zeitweise auftretendes Seitenstechen. Es lassen sich dann immer auch kleine, pleüritische Randgeräusche nachweisen, worauf ich noch später eingehen will.

Störungender Stimmbänderfunktion, die sich durch leichte Ermüdbarkeit und Mattigkeit der Stimme bis Heiserkeit äussern, wie gerade auch von Brecke (l. c.) beschrieben worden sind, können sowohl durch endothorakale Drüsenschwellungen als auch durch Infiltration der Lungenspitzen hervorgerufen werden.

In all diesen Fällen wird oft über eine Steigerǔng der erwähnten Beschwerden im Anschluss an probatorische Tuberkulininjektionen oder auch bei therapeutischen Tuberkulininjektionen in der Reaktionsperiode und bei weiblichen Patienten vor der Menstruation geklagt. Auch bei unabhängig von den angegebenen Momenten auftretenden Temperatursteigerungen werden gelegentlich wohl als Ausdruck stärkerer Schwellungszustände der Drüsen die erwähnten Beschwerden insbesondere von seiten des Magens angegeben.

Bei der objektiven Untersuchung fällt oft eine aus gesprochene Blässe der Ha u tauf, ohne dass Oligochromämie vorliegt. Es kann auch zu ausgesprochen lymphatischem Aussehen bei solchen Kranken kommen.

Manche derartige Patienten zeigen eine starke Gefässerregbarkeit, die sich durch rasch wechselnde Gesichtsfarbe äussert. Auch ungleichmässige Rötung der Wangen, sowie Ungleichheit der Pupillen kommt bei Fällen von reiner endothorakaler Drüsentuberkulose vor. Auffallend war bei einer Patientin das Auftreten einer zirkumskripten Rötung im II. Interkostalraum, rechts neben Sternum einige Tage vor der Periode, wobei dann auch in der ersten Zeit der Beobachtung die Temperatur jeweils anstieg.

Oft findet man gleichzeitig eine Vergrös serung der Halslymphdrüsen, die besonders bedeutsam erscheint, wenn die Drüsenschwellungen auf der einen Halsseite stärker ausgesprochen sind, als auf der anderen.

Manchmal sind auch diese vergrösserten Halsdrüsen etwas druckempfindlich und können sowohl prämenstruell als auch im An- 
schluss an Tuberkulininjektionen anschwellen; auch die übrigen Lymphdrüsen, einschliesslich der Tonsillen und Rachendachmandeln, können vorübergehend oder dauernd vergrössert sein. Bei einer zirka 30 jährigen Patientin mit einem fast reinen endothorakalen Drüsenprozess fanden sich abnorm grosse Tonsillenschwellungen, die sich in der Mitte beinahe berührten und operativ entfernt werden mussten.

Die von Petruschky angegebene Spinalgie der oberen Brustwirbeldornfortsätze (zwischen 2. und 7.) fand ich bei Erwachsenen nicht in der Häufigkeit wie B recke (l. c.); ebensowenig die Druckempfindlichkeit der oberen sternalen und paravertebralen Partien entsprechend den Dämpfungen - im ganzen aber doch nicht selten.

Die ösophageale Untersuchung nach $\mathrm{N}$ eisser (12) haben wir als zu umständlich nicht nachgeprüft.

Diagnostisch verwertbare Besonderheiten des Pulses konnten wir nicht beobachten.

Von wesentlicher, geradezu hauptsächlicher Bedeutung erscheinen mir die Ergebnisse der Perkussion, wie es in letzter Zeit auch von $\mathrm{Kr}$ äm er (l. c.) betont wird. Im Gegensatz hierzu hatte seinerzeit Sciallero (zit. bei Köhler l. c.) behauptet, dass bei tiefer gelegenem Sitz des fraglichen Leidens Perkussion und Auskultation regelmässig versagten, und auch $\mathrm{K}$ ö h l e r (l. c.) ist der Meinung, dass die geringeren und mittleren Schwellungen (von etwa Walnússgrösse) der betreffenden Lymphoglandulae weder mit Hilfo der Perkussion noch der Auskultation ausfindig gemacht werden können. Dass man bei genügender Beherrschung dieser betreffenden Untersuchungsmethoden sehr wohl in den meisten Fällen die Diagnose von endothorakalen Drüsen- resp. Hilusprozessen stellen kann, werden. wir später sehen. Wie erwähnt, ist eine gewisse Übung und Erfahrung in dieser Untersuchungsmethode zur annähernden Diagnosenstellung durchaus notwendig, und zwar ist die abgestufte. Perkussion, wie sie von Ebstein (13) und Turban (14) gelehrt wird, von besonderem Wert. Gerade die Tastperkussion und die direkte Perkussion sind zur Auffindung von etwas tiefer liegenden Dämpfungen unerlässlich. Turban (l. c.) erwähnt schon, dass es ihm mit Ebstein scher Perkussion gelungen ist, besonders bei Kindern parasternale resp. paravertebrale Drüsendämpfungen festzustellen und dass ihm die Bestätigung dèr Diagnose - Drüsenschwellungen - durch die Autopsio nieht fehlte.

Wenn man aber orst einmal gelernt hat, seine Aufmerksamkeit auf die Hilusgegend zu-lenken, wird man erstaunt sein, wie häufig 
man Dämpfungen dort findet. In dieser Hinsicht verdanken wir der Röntgenphotographie wichtige Hinweise.

Bei Gesunden sind diese Hilusdämpfungen nicht vorhanden, was ich wiederholt festgestellt habe. Es sei dies nur deshalb erwähnt, weil man annehmen könnte, dass die im Hilus zusammenlaufenden grossen Gefässe und Bronchien in Verbindung mit den rormalgrossen Hilusdrüsen schon eine physiologische Dämpfung hervorbringen könnten.

Von Bedeutung ist der Umstand, dass die endothorakalen Drüsendämpfungen weiter untenliegen als die eigentlichen Spitzendämpfungen. Für die vorderen Dämpfungen kommen mehr die Mediastinaldrüsen, für die hinteren die Hilusdrüsen in Betracht. Vorne sind die eigentlichen Drüsendämpfungen am deutlichsten ausgesprochen in den dem II. und auch wohl dem III. Interkostalraum angehörigen parasternalen Partien. Die sehr häufigen, oft die Spitzendämpfungen übertreffenden parasternalen Dämpfungen im I. Interkostalraum sind wenigstens bei Erwachsenen öfters auf Verbreiterungen und Verdickungen der sternalen Partien der Klavikula und der ersten Rippe, sowie Retraktion der sternalen Lungenränder zurückzuführen, als auf Vergrösserungen der entsprechenden Mediastinaldrüsen, wie dies D'Espine (14), Bandelier und Roepke (l. c.) annehmen. Hingegen findet man nicht selten in Fällen starker Mediastinaldrüsenschwellungen auch deutliche Schallverkürzungen über den oberen Partien des Sternums.

Wie erwähnt, machen sich die Hilusdrüsenvergrösserungen mehr durch paravertebrale Dämpfungen bemerkbar, worauf schon Biedert (l. c.), Turban (l. c.), Brecke (l. c.) und besonders $\mathrm{Kr}$ ämer (l. c.) aufmerksam gemacht haben. Am leichtesten ist der Nachweis dieser. Dämpfungen, die etwa der Höhe des 3 . bis 5. Brustwirbels oder mit anderen Worten der Mitte der Skapula entsprechen, wenn keine oder eine durch eine sonore Zone davon abgegrenzte Spitzendämpfung vorhanden ist, was Krämer auch schon beobachtet hat.

Meist findet man bei den so überaus häufigen ${ }^{1}$ ) sleichzeitig vorhandenen infiltrativen Prozessen der oberen Lungenpartien eine stärkere, meist auch paravertebral am deutlichsten ausgesprochene Dämpfung der Lungenspitzen, die sich gegen oder auch etwas unterhalb Spina scapulae ein wenig aufhellt und dann gegen Mitte Scapulae

1) H. J. Bing schätzt die Häufigkeit der durch Bronchialdrüsenschwellungen hervorgerufenen Dämpfungen bei Lungenleidenden auf $75 \%$ der Fälle. 
wieder stärker wird. Oft können diese geringeren subspinalen Dämpfungen im Laufe einer längeren Behandlung dann fast völlig oder vollständig verschwinden und der oben erwähnten sonoren.Zone Platz machen. Die eigentlichen Hilusdämpfungen bleiben immer bestehen, wenn auch oft in geringerem Grade wie zu Anfang einer Kur. Meist ragen diese eigentlichen Hilusdämpfungen nach aussen zu nicht über die Breite von zwei Querfingern, gehen also selten bis zum inneren Skapularand, vorausgesetzt, dass man den Patienten eine gebeugte Haltung mit gekreuzten Armen einnehmen lässt.

Über diesen gedämpften Gebieten lässt sich auch immer eine Resistenzvermehrung mit Ebsteinscher und direkter Perkussion feststellen.

Wichtig ist, die Perkussion in allen vier Hauptrichtungen vorzunehmen, also nicht nur von oben nach unten, sondern auch von unten nach oben, von aussen nach innen und von innen nach aussen; die dabei gefundenen Grenzen müssen sich natürlich decken.

Doppelseitige Hilusdämpfungen können, wie dies sogar in der Mehrzahl der Fälle zutrifft, bezüglich Intensität und Extensität ganz verschieden sein, worauf auch $\mathrm{Kr}$ äm e r (l. c.) aufmerksam macht; in solchen Fällen fanden wir fast immer eine Übereinstimmung der Perkussionsergebnisse mit den röntgehologisch gewonnenen Drüsenschatten, ein fernerer Beweis für den Wert einer ausgebildeten Perkussionstechnik.

In ausgesprochenen Fällen, besonders auch ron doppelseitigen Hilusdämpfungen, fand ich auch jeweils die pon Kor a n y i (17) angegebene und von $\mathrm{De} \mathrm{la} \mathrm{Camp} \mathrm{(l.} \mathrm{c.)} \mathrm{und} \mathrm{Mieczylaw} \mathrm{Micha-}$ low icz (18) bestätigte Schallverkürzung bei Perkussion der betreffenden Rückenwirbeldornfortsätze. Wichtig ist auch, hierbei ein schmales Plessimeter zu benutzen und nur leise zu perkutieren.

Dass diese Hilusdämpfungen nicht nur durch Drüsenvergrösserungen hervorgerufen werden, hat auch schon $\mathrm{Kräm}$ er (l. c.) angenommen. Dieser Autor glaubt, dass durch die vergrösserten Drüsén eine Stauung im Lymphgefässsystem der betreffenden Gegend hervorgerufen werde, eine Auffassung, der ich mich in dieser Form aus verschiedenen Gründen nicht anschliessen möchte.

Viel wahrscheinlicher erscheint mir die Annahme, dass in der Umgebung der vergrösserten aktiven Drüsen ein entzündliches Infiltrat entstehen kann, wie es z. B. "H ug u enin (l. c.) beschreibt, ganz abgesehen davon, dass bei derartigen Drüsenprozessen oft gleichzeitig eine strahlenförmig, gegen den Hilus zu konfluierende Peribronchitis besteht, wodurch die Hilusdämpfung vergrössert und 
verstärkt wird. In gleichem Sinne kann auch eine durch die Drüsenvergrösserung erzeugte Atelektase des umgebenden Lungengewebes wirken. Gerade diese sekundären Dämpfungen sigd es, die im Laufe einer Behandlung, insbesondere einer vorsichtigen Tuberkulinkur, verschwinden und der erwähnten sonoren Zone Platz machen können, wodurch die gesamte Hilusdämpfung an Intensität und Extensität abnimmt Rein klinisch lassen sich die endothorakalen Drüsenschwellungen von den, wenigstens bei Erwachsenen, sie wohl fast immer umgebenden übrigen Hilusprozessen nicht tremnen.

Wenn auch die auskultatorischen Phänomene nicht so charakteristisch sind wie die perkutorischen, so möchte ich sie doch nicht so ganz beiseite stellen, wie dies $\mathrm{Kräm}$ er (l. c.) tut. Schon Biedert (l: c.) erwähnt, dass in den durch die Drüsenschwellungen erzeugten gedämpften Partien die A tm ung of $\mathrm{t}$ abge s chwä cht und un rein sei, bei Kindern soll nach $\mathrm{H}$ e u b n e r (1. c.) das Atmen oft einen lauten Trachealcharakter annehmen können. Ich konnte diese Erscheinung bei Erwachsenen nie feststellen, doch kann es je nach dem Grad der Verdichtung des Hilusgewebes zu einem nach dem bronchialen $z u$ veränderten Atmen kommen. Häufig ist das Inspirium verschärft, das Exspirium verlängert; noch charakteristischer ist ein ausgesprochen keuchendes Geräusch, das meist nur bei stärkerem Husten, wobei der Patient den Mund weit öfnen soll, zu hören ist, und sich von dem mehr konstanten Keuchen, wie es in vernarbten und geschrumpften Spitzen unter Umständen zu hören ist, dadurch unterscheidet, dass es je nach dem Schwellungszustand der Drüsen verändert wird.

Auck.wechselnde bronchitische Geräusche, Pfeifen oder Giemen konnte ich zur Zeit stärkerer Drüsenschwellung nachweisen.

Dass je nach der Stärke der Dämpfung stimmfremitus und Bronehophonie mehr oder weniger verstärkt sind, ist ja nicht weiter merkwürdig; in einigen Fällen konnte ich die Angaben $\mathrm{De}$ la Camps (l. c.) bestätigen, dass der Stimmfremitus abgeschwächt, dagegen die Bronchophonie verstärkt war.

Besonders empfehlenswert ist die Anwendung der Flüster stimme bei der Prüfung der Bronchophonie, die bei stärkeren Hilusdämpfungen einen eigentümlich $\mathrm{h}$ a u ch en den bis keuchenden, sehr nah ins Ohr klingenden Charakter annehmen kann.

Eines Phänomens ist noch zu gedenken, das indirekt in Beziehung zur endothorakalen Drüsentuberkulose steht, und gerade auch 
im Anschluss an Zwischeniniektionen, reaktive Tuberkulininjektioner, sowie prämenstruell nicht selten auftritt. Es ist dies ein meist rasch wieder vor übergehendes feines Pleurareiben in den unteren Lungenrändern, gewöhnlich am deutlichsten in der unteren Axillargegend. Diese Erscheinung sah ich besonders häufig in Fällen auftreten, in denen das Röntgenbild neben deutlichen Drüsenvergrösserungen ausgesprochene peribronchitische Veränderungen zeigte.

Auch $\mathrm{Hu}$ g u en in (1. c. 7) hat auf diesen direkten Zusammenhang zwischen diesen Randpleuritiden und Bronchialdrüsentuberkulose hingewiesen.

Eill weiteres von Brecke (l. c.) erwähntes Symptom: Un reinigkeiten über den Klappen der grossen Gefässe, konnte ich nur selten bestätigen. Hingegen fand ich bei stärker ausgesprochenen einseitigen parasternalen Dämpfungen, seien sie nun durch Mediastinaldrüsenschwellungen oder Infiltrate der betreffenden oberen Partien hervorgerufen, eine de utliche Akzen tu i erung des zweiten Ton es, und zwar beirechtsseitigen Prozessen über der A orta, bei linksseitigen über der Ar. teria pulmonalis.

Toxische Albuminurie, wie sie in solchen Fällen bei Kindern nach Huguenin (1. c. 8) vorkommen soll, habe ich bei Erwachsenen nie beobachtet.

Von grosser diagnostischer Bedeutung ist die Beobachtung der Temperaturverhältnisse.

Schon von De la Camp (1. c.), Huguenin (l. c. 8) und anderen wird auf geringe, schwankende Temperaturerhebungen bei diesen Patienten hingewiesen, die oft monate- und jahrelang dauerten. In vielen derartigen Fällen meiner Beobachtung traten kleine Temperaturerhebungen, deren Maximum fast immer unter $38^{\circ} \mathrm{lag}$, in direkt mehr oder weniger regelmässig intermittierenden Perioden auf, ersteres besonders bei Frauen vor der Periode.

Auch sonst ist die Temperatur bei diesen Patienten sehrlabil; oft genügt ein kleiner Schnupfen, eine grössere körperliche Anstrengung, eine zu grosse Tuberkulindosis, um wieder eine längere Fieberperiode hervorzurufen.

Beiläufig bemerkt ist es mir in der grössten Mehrzahl dieser Fälle gelungen, eine dauernde Entfieberung durch kleinste Tuberkulindosen zu erzielen, selbst in einigen ausserordentlich hartnäckigen Fällen (19).

Immerhin ist die Entfieberung schwieriger zu erzielen und nimmt längere Zeit in Anspruch als in Fällen reiner Lungentuberkulose, worauf ich an anderer Stelle (l. c.) schon hingewiesen habe. 
Dabei soll nicht verschwiegen werden, dass solche Patienten mit langer Fieberdauer auch, obgleich selten, spontan entfiebern können. Andererseits laufen sie immer Gefahr, dass der tuberkulöse Prozess von den Drüsen direkt und auf dem Lymphwege in die Lungen oder auf dem Blutwege in andere Organe dringt.

Im ersteren Falle bilden sich dann die bekannten, auf dem Röntgenphotogramm so schön zur Darstellung gelangenden strahligen peribronchitischen Hilusfiltrate, die dann auch, zu einer entsprechenden Verstärkung der übrigen klinischen Symptome, insbesondere der Dämpfungen führen.

Bei Erwachsenen haben allerdings diese Drüsenprozesse, so hartnäckig sie sein können - mir sind Fälle von 2--3 jähriger Dauer bekannt -, meist einen gutartigen Charakter:

Es ist wohl nicht zuviel gesagt, wenn weitaus die meisten Fälle von chronischer und intermittierender Subfebrilität bei sonst negativem klinischem Befunde - besonders wenn etwa tuberkulöse Antezedenzien in der Familie oder bei den Patienten selbst vorliegen - auf solche latenten Drüsenprozesse bezogen werden.

Sollten die erwähnten gewöhnlichen klinischen Untersuchungen kein genügend klares Bild geben, so müssen noch die folgenden Untersuchungsmethoden herangezogen werden, wie dies in allen derartigen Fällen zur Klärung der Diagnose durchaus wünsehenswert ist.

Da sind zunächst die spezifis chen Proben zur Gewinnung einer Anschauung über die Ahtivität der Drüsen- resp. Hilusprozesse von Wichtigkeit.

Zunächst ist es zu empfehIen, mit abgestuften Tuberkulinlösungen, begimnend mit $1-, 21 / 2^{-}, 5 \%$ iger usw. Lösung,- wie sie schon von verschiedenen Autoren und auch von uns längere Zeit rerwendet werden, Pir qu et sche Reaktionen zu màchen. Eine Reaktioli auf schwache Lösungen weist nach unseren zahlreichen Erfahrungen mit grosser Wahrscheinlichkeit auf die Gegenwart eines aktiven Prozesses hin, handle es sich nun um reine Drüsen- oder um Lungentuberkulose oder beides.

Vor der alten Probeinjektion kann meist Umgang genommen werden, schon mit Rücksicht auf die Gefahr der Hervorrufung einès chronischen subfebrilen Zustandes; eine Möglichkeit, die trotz aller Widerreden vorhanden ist, besonders bei diesen so temperaturlabilen Drüsenkranken.

Höchstens kann etwa die Löw enstein sche Modifikation versucht werden, unter genauer Beobachtung der Herdreaktion (Ver- 
stärkung der Dämpfung, Auftreten von Keuchen und bronchitischen Geräuschen und pleuritischen Phänomenen, die vorher nicht oder nur schwach ausgesprochen vorhanden waren).

In Fällen reiner Drüsentuberkulose dürfen natürlich die Lungenspitzen selbst keine reaktiven Erscheinungen aufweisen; hingegen finden wir, falls es sich um aktive Drüsenprozesse handelt, die Veränderungen in der Blutzusammensetzung, wie sie in der folgenden Arbeit von Schulz beschrieben sind.

Bezüglich der so wichtigen röntgenologischen Untersuchungen muss zunächst mit $\mathrm{K} \ddot{o} \mathrm{~h} l$ e r (l. c.) und $\mathrm{D}$ e 1 a $\mathrm{C}$ a m p (l. c.) darauf hingewiesen werden, dass normale Drüsen keine, markig geschwollene Drüsen nicht immer, und nur verkalkte und bindegewebig veränderte Drüsen gute Schatten geben.

Es kann also vorkommen, dass aktive Drüsenprozesse, die nur zu markigen Veränderungen geführt haben, röntgenologisch nichts ergeben, während die übrigen klinischen Erscheinungen, inklusive der Blutuntersuchungen, auf einen aktiven Drüsenprozess hinweisen.

Immerhin kommt dieser negative Ausfall bei Erwachsenen, bei denen es sich ja wohl meist um ein Rezidiv eines alten Prozesses, der zum Teil schon regressive, schattenwerfende Veränderungen gemacht hat, handelt, selten vor. Jedenfalls konnten wir in unseren Fällen im mer mehr oder weniger deutlich ausgesprochene Drüsenschatten nachweisen.

Auf die genauere Beschreibung der Drüsenschatten kann ich verzichten, da dies von Köhler (l. c.), Ri eder (20), D e la Camp (l. c.) sowie anderen Autoren geschehen ist.

Es sei nur an die projektilartigen Bilder, die im ganzen schärfere Grenzen zeigen als die eigentlichen Lungenherde, erinnert, die von diesen Drüsen geliefert werden. Meist strahlen von den eigentlichen Drüsenschatten baumartig verzweigte Linien oder Kettenbildungen

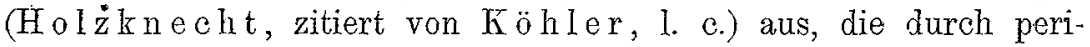
bronchitische Stränge hervorgerufen werden.

Beiläufig bemerkt machen wir unsere Röntgenaufnahmen unter Verwendung des ausserordentlich praktischen Drehstuhls von $\mathrm{Rze}$ w uski Davos-Platz in dorsoventraler Richtung nach Angabe von Rieder (1. c.). Es sei auch àuf die besonders schönen Reproduktionen iil der Arbeit dieses Autors hingewiesen.

Wichtig ist bei den Röntgenaufnahmen auch die Beobachtung von Zug und Druckerscheinungen der vergrösserten Drüsen auf die Trachea und die Bronchien.

Ein kurzes Resumé meiner und der Schulzschen Beobachtungen lasse ich nach der zweiten Arbeit folgen, und füge zuletzt 
noch als Paradigmata sechs Auszüge aus einschlägigen Krankengeschichten, nebst Kopien der dazu gehörigen Röntgenogramme bei.

\section{Literatur.}

1. Biedert,Ph. u. Litting, L., Die Krankheiten der Bronchialdrüsen. Festschrift zu Hennochs 70. Geburtstag.

2. Heubner, Lehrbuch der Kinderheilkunde.

3. De la Camp, Die klinische Diagnose der Bronchialdrüsentuberkulose. Ergebnisse d. inn. Med. u. Kinderheilk. Bd. I.

4. Brecke, Zur Dingnose der Schwellungen der endothorakalen Lymphdrüsen. Beitr. z. Klinik d. Trib. Bd. IX. Heft 3.

5. Köhler, Zur Röntgendiagnostik der kindlichen Lungendrüsentuberkulose. Hamburg. Lukas Gräfe \&-Silben. 1906.

6. Krämer, Bronchialdrüsen-Dämpfungen im Interskapularraum und ihre Bedeutung für die Diagnose und spezif. Therapie der Tuberkulose. Beitr. zur Klinik d. Tub. Bd. XIV. Heft 3.

7. Huguenin, Bronchialdrüsentuberkulose und Lungenerkrankungen. Korr.Blatt f. Schweiz. Ärzte. XL. Jahrg. 1910. Heft 4-6.

8. Derselbe, Prädisposition der Lungenspitzen u. Inhalationstub. Korr.-Blatt f. Schweiz. Ärzte. XLI. Jahrg. 1911. Nr. 5-8.

9. Dautwitz, Über Diagnose und spezif. Behandlang der latenten endothorakalen Drüsentuberkulose des kindlichen Alters. Beiheft zar Medizin. Klinik. No. 9. 1908.

10. Bandelier und Roepke, Die Klinik der Tuberkulose. Würzburg 1911. Cart Kabitzsch.

11. Heymann, Versuche an Meerschweinchen über die Aufnahme inhalierter Tuberkelbazillen in die Lunge. Zeitschr. f. Hyg. und Infektionskrankheiten. 60 Bd. 1908.

12. Neis ser, E., Über Sondenpalpation der Bronchialdrüsen bei gewissen leichtesten Formen der Tub. Deutsches Archiv f. klin. Med. 1905. Bd. 86.

13. Ebstein, W., Die Tastperkussion. Verlag F. Enke, Stuttgart 1901.

14. Turban, K., Beiträge zur Kenntnis der Langentuberkulose. Verlag J. F. Bergmann, Wiesbaden 1899.

15. D'Espine, Diagnostic précore de la Tuberc. des ganglions bronchiques chez les enfants. Tuberculosis. Mai 1907.

16. Bing, H., Über die Diagnose der Bronchialdrüsen-Anschwellung. Ugeskrift for Laeger. Nr. 36. 1910.

17. Koranyi, Zeitschr. f. klin. Med. 60. 1906. Heft 3 u. 4.

18. Mieczylaw Michal ow icz, Über die Verwertung der Wirbelsäuleperkussion bei der Diagnose der Tracheobronchialdrüsentuberkulose im Kindesalter. Jahrb. f. Kinderheilk. 1910. 71. p. 27.

19. Philippi, Über Entfieberungen bei Lungentuberkalose durch kleinste Dosen Tuberkulin. Beitr. z. Klinik d. Tub. Bd. XVI. Heft 3.

20. Rieder, Zur Röntgendiagnostik bei Anfangstuberkulose der Lungen. Beitr. z. Klinik d. Tub. Bd. XII. Heft 2. 